\title{
Histological Remission in Ulcerative Colitis in Deep Remission under Treatment with Adalimumab
}

\author{
Antonio Tursi ${ }^{1,2}$, Rosanna Nenna ${ }^{3}$, Giovanni Musci ${ }^{3}$, Walter Elisei ${ }^{4}$, Marcello Picchio ${ }^{5}$
}

1) Territorial Gastroenterology Service, ASL BAT, Andria (BT);

2) Department of Medical and Surgical Sciences, Postgraduate School of Digestive Diseases, Catholic University, Rome;

3) Department of Pathology, Lorenzo Bonomo Hospital, Andria (BT);

4) Division of

Gastroenterology, S. Camillo Hospital, Roma;

5) Division of Surgery, P. Colombo Hospital, Velletri Roma; Italy

\author{
Address for correspondence: \\ Antonio Tursi, MD \\ Via Torino, 49 \\ 76123 Andria (BT), Italy \\ antotursi@tiscali.it \\ ORCID: http://orcid.org/0000- \\ 0001-5767-5541
}

\begin{abstract}
Background \& Aims: Histological remission (HR) has been recently demonstrated as the last therapeutic goal in ulcerative colitis (UC), but it is unknown whether and how it may occur. Our aim was to assess the histology during the follow-up of an UC population in deep remission under treatment with adalimumab (ADA). Methods: We performed a retrospective study on 22 UC patients who were in deep remission and followed-up while receiving therapy with ADA. Colonoscopy in those patients was performed every year. Four-quadrant biopsies every $10 \mathrm{~cm}$ were obtained during each colonoscopy and assessed by hematoxylin and eosin stain. Histological activity was classified using the Geboes scale.

Results: A total of 22 patients were enrolled in the study. The mean follow-up of those patients was $28 \pm 7$ months, and 2,592 biopsy specimens in total were taken during 108 colonoscopies performed during the follow-up. At the beginning of the follow-up, histological inflammation was found in 15/22 (68.2\%) of patients in deep remissio while receiving maintenance ADA therapy, 8/22 (36.4\%) of them with Geboes score $\geq 3.1$. At the end of the follow-up, when patients were still in deep remission while receiving maintenance ADA therapy, only 4 patients (18.2\%) had at least one biopsy specimen with evidence of any histological inflammation during the follow-up; only two patients $(9.1 \%)$ had Geboes score $\geq 3.1$.

Conclusions: Our study shows for the first time that UC patients in deep remission under ADA may reach HR, but it seems slower than other clinical or endoscopic goals.
\end{abstract}

Key words: deep remission - histological remission - adalimumab - ulcerative colitis.

Abbreviations: ADA: adalimumab; HR: histological remission; IFX: infliximab; UC: ulcerative colitis; TNF: tumor necrosing factor.

\section{INTRODUCTION}

Histological remission (HR) has been recently pointed out as the last therapeutic goal in ulcerative colitis (UC) [1-3]. Although there is no consensus yet about the best histological index to use in real life $[2,4,5]$, histological features seem to be a reliable predictor of disease outcomes after therapy, and HR seems to be the new frontier in the treatment of UC [1-3]. In fact, a recent meta-analysis. found that histologic and endoscopic remission correlated strongly, and less severe clinical disease activity, and topic 5 amino-salicylates and corticosteroid use were associated with higher HR rates [3].

Reliable literature data regarding HR under treatment with immunosuppressors or biologics are still lacking. However, HR under treatment with anti-tumor necrosing factor (TNF) $a$ is not so promising. We previously failed to find a significant correlation between endoscopic and histological activity in UC under treatment with Infliximab (IFX) [6], and two recent studies failed to find a significant relationship between endoscopic and histologic response to adalimumab (ADA) in UC patients $[7,8]$. However, we do not know whether and how the histologic inflammation in UC may change during deep remission under ADA. This considered, we assessed the histology during the follow-up of an UC population in deep remission under treatment with ADA.

\section{METHODS}

We performed a retrospective study on 22 UC patients who were in deep remission and followed-up while receiving therapy 
with ADA. Deep remission in UC is defined as the reaching of clinical and endoscopic remission [9]. To be considered "in remission" for enrolment in this study, participants had to have Mayo stool frequency subscore of 0 or 1 , Mayo rectal bleeding subscore of 0 , and Mayo subscore for endoscopy $\leq 1$ for at least 6 months and have had no changes in their UC medications (ADA) or any steroid use in the prior 6 months. Thus, we defined it as Mayo score $\leq 2$ and Mayo subscore $\leq 1$.

According to our standard procedure, colonoscopy in UC patients in deep remission under biologics was performed every year. During colonoscopy the colon was divided into 6 segments (cecum, ascending colon, transverse colon, descending colon, sigmoid colon, and rectum). Four-quadrant biopsies every $10 \mathrm{~cm}$ were obtained during each colonoscopy and assessed by hematoxylin and eosin stain. Histological activity in all segments was classified using the Geboes scale [10] by two pathologists (R.N. and G.M.), blinded to the patient's disease status and endoscopic score. Histological remission was considered when Geboes score was $<1$.

The collection and analysis of data were performed using MedCalc Release 7.3.0.1. Interobserver agreement was assessed by weighted kappa value and was classified as follows: poor, $0-0.20$; fair, $0.21-0.40$; moderate, $0.41-0.60$; good, 0.61-0.80; and excellent, 0.81-1.00. Fisher's exact test was used for categorical variables. The level of significance was $\mathrm{p}=0.05$.

\section{RESULTS}

A total of 22 patients were retrospectively enrolled in the study (Table I). All patients were in deep remission at the beginning of the study; all were under treatment with ADA and took also 5 amino-salicylates orally. The mean follow-up of those patients in deep remission was $28 \pm 7$ months. The mean number of biopsy specimens per colonoscopy was 24 , and 2,592 biopsy specimens in total were taken during 108 colonoscopies performed during the follow-up.

All patients were under clinical and endoscopic remission during the follow-up, no change in treatment was performed during the follow-up (namely all patients continued to take $\mathrm{ADA}$ and 5 amino-salicylates at the same dose during the follow-up), and none of them were withdrawn during the follow-up.

The concordance between the two histopathologists in assessing inflammatory infiltrate was excellent (weighted kappa $=0.89$ ). The presence (Geboes score $\geq 1$ ) and abnormality (Geboes score $\geq 3.1$ ) of histological inflammation at the beginning and at the end of follow-up are reported in Fig. 1. At the beginning of the follow-up, histological features of inflammation were found in 15/22 (68.2\%) of patients in deep remission while receiving maintenance ADA therapy, $8 / 22(36.4 \%)$ of them with Geboes score $\geq 3.1$. At the end of the follow-up, when patients were still in deep remission while receiving maintenance ADA therapy, only 4 patients $(18.2 \%)$ had at least one biopsy specimen with evidence of any histological inflammation during the follow-up; only two patients $(9.1 \%)$ had Geboes score $\geq 3.1$, in both cases detected in the rectum (1 patient affected by pancolitis and 1 patient affected by distal colitis) (Table II).
Table I. Characteristics of the study group.

\begin{tabular}{ll}
\hline Males/Females & $10 / 12$ \\
Indications for ADA treatment & \\
$\quad$ Steroid-dependent UC & $15(68.1)$ \\
$\quad$ Steroid-resistant UC & $7(31.9)$ \\
Mean (range) age (years) at the time of diagnosis & $27.3(18-49)$ \\
Mean (range) age (years) at the time of the first ADA & $29.20(24-55)$ \\
infusion & \\
$\begin{array}{l}\text { Mean (range) duration (years) of the disease prior to } \\
\text { ADA treatment }\end{array}$ & $6(1-8)$ \\
$\begin{array}{l}\text { Mean (range) follow-up (months) under ADA } \\
\text { treatment }\end{array}$ & $34(12-44)$ \\
Disease's localization & \\
$\quad$ Distal colitis & $1(4.5)$ \\
$\quad$ Left-sided colitis & $9(40.9)$ \\
$\quad$ Pancolitis & $12(54.6)$ \\
Smokers & $8(36.4)$ \\
Associated drugs & \\
5ean Amino-salicylates & \\
Mean (range) Mayo score at the beginning of the study & $1(0-1)$ \\
study (mg/kg) & $1.3(0.5-4.8)$ \\
\hline
\end{tabular}

Values are expressed as number (percentage), unless otherwise specified. ADA: adalimumab; CRP: C reactive protein.

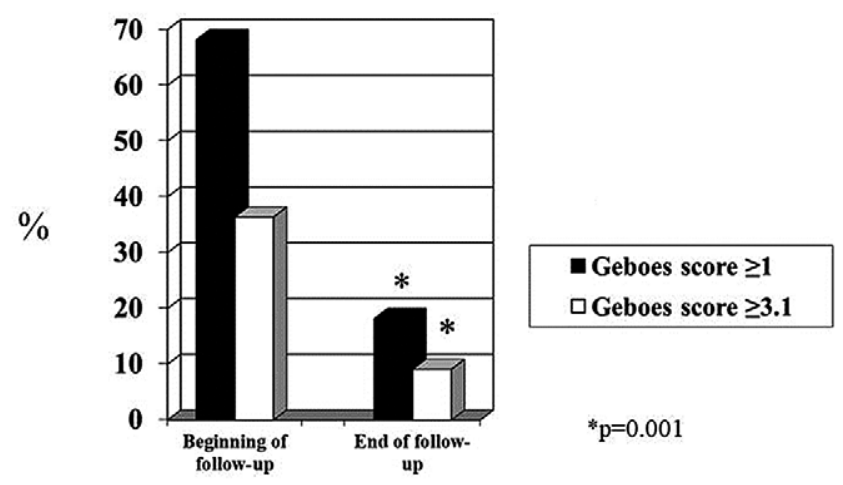

Fig. 1. Bar graphic showing the histological inflammation subdivided according to the Geboes score $\geq 0.1$ and the Geboes score $\geq 3.1$ at the beginning and at the end of follow-up ( $\mathrm{p}=0.001$, Fisher's exact test).

\section{DISCUSSION}

Histological remission is becoming an important therapeutic goal because it is associated with lower disease relapse among UC patients reaching endoscopic remission [11].

Data about the HR when using IFX are not so promising. D'haens et al. [12] found that architectural alteration persisted in all adult UC patients reaching endoscopic remission under IFX, while significantly better results were obtained in pediatric UC patients [13]. Finally, we found that adult UC patients in deep remission under treatment with IFX failed to have a significant correlation with HR [6].

In this study we tried to observe what happens in UC patients in deep remission under ADA and receiving 
Table II. Histological inflammation subdivided according to the extent of the disease at the beginning and at the end of follow-up

\begin{tabular}{|c|c|c|c|c|c|c|}
\hline \multirow[t]{2}{*}{ Histological inflammation } & \multicolumn{2}{|c|}{ Pancolitis (12 pts) } & \multicolumn{2}{|c|}{ Left-sided colitis (9 pts) } & \multicolumn{2}{|c|}{ Distal colitis (1 pt) } \\
\hline & $\begin{array}{l}\text { Beginning of } \\
\text { follow-up }\end{array}$ & $\begin{array}{l}\text { End of } \\
\text { follow-up }\end{array}$ & $\begin{array}{l}\text { Beginning of } \\
\text { follow-up }\end{array}$ & $\begin{array}{l}\text { End of } \\
\text { follow-up }\end{array}$ & $\begin{array}{l}\text { Beginning } \\
\text { of follow-up }\end{array}$ & $\begin{array}{l}\text { End of } \\
\text { follow-up }\end{array}$ \\
\hline Geboes score $\geq 0.1$ & $8(66.6)$ & $2(16.6)$ & $6(66.6)$ & $1(11.1)$ & $1(100)$ & $1(100)$ \\
\hline Right colon & $8(66.6)$ & - & - & - & - & - \\
\hline Geboes score $\geq 0.1$ but $<3.1$ & $6(50)$ & - & - & - & - & - \\
\hline Geboes score $\geq 3.1$ & - & - & - & - & - & - \\
\hline Transverse & $6(60)$ & - & - & - & - & - \\
\hline Geboes score $\geq 0.1$ but $<3.1$ & $2(16.6)$ & - & - & - & - & - \\
\hline Geboes score $\geq 3.1$ & - & - & - & - & - & - \\
\hline Left sided colon & $4(33.3)$ & $1(8.3)$ & $6(66.6)$ & - & - & - \\
\hline Geboes score $\geq 0.1$ but $<3.1$ & $3(25)$ & $1(8.3)$ & $3(33.3)$ & - & - & - \\
\hline Geboes score $\geq 3.1$ & $1(8.3)$ & - & $1(11.1)$ & - & - & - \\
\hline Distal colon & $4(33.3)$ & $1(8.3)$ & $4(44.4)$ & $1(11.1)$ & $1(100)$ & $1(100)$ \\
\hline Geboes score $\geq 0.1$ but $<3.1$ & $3(25)$ & - & - & - & - & $1(100)$ \\
\hline Geboes score $\geq 3.1$ & $3(25)$ & $1(8.3)$ & $2(22.2)$ & $1(11.1)$ & $1(100)$ & - \\
\hline
\end{tabular}

Values are expressed as number percentage of patients (pts). ( $\mathrm{P}=0.001$, Fisher's exact test).

maintenance treatment with ADA. We found significantly better results than that obtained when using IFX, since almost $70 \%$ of UC patients in deep remission under ADA treatment reach $\mathrm{HR}$ during the follow-up. This finding differs significantly from that recently reported by other recent studies regarding the efficacy of ADA in obtaining HR. Fernández-Blanco et al. [7] found that HR (indicated by a Geboes score <3.0) was obtained in $31 \%$ of patients at $52^{\text {nd }}$ week of ADA treatment, with a fair relationship with mucosal healing. More recently, the VARSITY double-blind placebo-controlled trial found that $\mathrm{HR}$ at week 52 (indicated by a Geboes score $<2.0$ ), occurred in only $3.1 \%$ (12 of 386) of the patients treated with ADA [8]. One possible explanation is linked to the different criteria used to describe HR. Another hypothesis is that patients with deep remission under ADA may have higher chances to reach $\mathrm{HR}$. In fact, Yarur et al. [14] found that higher ADA levels were best associated with mucosal healing and HR [14]. Even if we did not assess ADA levels in our population, we can speculate that patients in deep remission may have higher ADA levels and therefore higher chances to reach HR.

This study confirms that HR is generally a slow cranialcaudal process also under treatment with $\mathrm{ADA}$, and that distal colon seems to be the most refractory colonic district to HR. It has been recently reported that segmental HR in a proximal-to-distal direction occurs in the large majority of UC patients reaching HR [15], and the same process seems to occur also in UC under IFX in UC. We found that TNFa levels in rectal mucosa were not reduced despite clinical and endoscopic response to IFX [16]. Why this occurs is unknown. Leal et al. [17] found that anti-TNFa therapy significantly downregulated a subset of inflammatory genes even in patients who failed to achieve endoscopic remission. This probably means that other inflammatory ways have to be considered in UC patients in deep remission but with persistence of histological inflammation.
This study has clear limitations: the absence of the standard system for grading of histological activity, the retrospective design, the small population assessed.

\section{CONCLUSIONS}

Our study shows for the first time that UC patients in deep remission under ADA may reach $\mathrm{HR}$, but it seems to be slower to achieve than other clinical or endoscopic goals. Further, prospective studies have to confirm these results and to determine the impact of HR in medium- and long-term clinical outcomes.

\section{Conflicts of interest: None to declare.}

Authors' contribution: A.T. conceived and designed the study. A.T., R.N., G.M., W.E., M.P. collected and analyzed the data, drafted the manuscript and critically revised it and approved the final version.

\section{REFERENCES}

1. Long MD, Rubin DT. Histologic Remission in Ulcerative Colitis: Are We There Yet? Am J Gastroenterol 2019;114:713-715. doi:10.14309/ ajg.0000000000000235

2. Chateau T, Feakins R, Marchal-Bressenot A, Magro F, Danese S, Peyrin-Biroulet L. Histological Remission in Ulcerative Colitis: Under the Microscope Is the Cure. Am J Gastroenterol 2020;115:179-189. doi:10.14309/ajg.0000000000000437

3. Battat R, Duijvestein M, Guizzetti L, et al. Histologic Healing Rates of Medical Therapies for Ulcerative Colitis:A Systematic Review and Meta-Analysis of Randomized Controlled Trials. Am J Gastroenterol 2019;114:733-745. doi:10.14309/ajg.0000000000000111

4. Magro F, Langner C, Driessen A, et al; European Society of Pathology (ESP); European Crohn's and Colitis Organisation (ECCO). European 
consensus on the histopathology of inflammatory bowel disease. J Crohns Colitis 2013;7:827-851. doi:10.1016/j.crohns.2013.06.001

5. Magro F, Lopes J, Borralho P, et al; Portuguese IBD Study Group [GEDII]. Comparison of the Nancy Index With Continuous Geboes Score: Histological Remission and Response in Ulcerative Colitis. J Crohns Colitis 2020;14:1021-1025. doi:10.1093/ecco-jcc/jjaa010

6. Tursi A, Elisei W, Picchio M, et al. Histological inflammation in ulcerative colitis in deep remission under treatment with infliximab. Clin Res Hepatol Gastroenterol 2015;39:107-113. doi:10.1016/j.clinre.2014.07.012

7. Fernández-Blanco JI, Fernández-Díaz G, Cara C, Vera MI, Olivares D, Taxonera C. Adalimumab for Induction of Histological Remission in Moderately to Severely Active Ulcerative Colitis. Dig Dis Sci 2018;63:731-737. doi:10.1007/s10620-018-4935-5

8. Sands BE, Peyrin-Biroulet L, Loftus EV Jr, et al; VARSITY Study Group. Vedolizumab versus Adalimumab for Moderate-to-Severe Ulcerative Colitis. N Engl J Med 2019;381:1215-1226. doi:10.1056/ NEJMoa1905725

9. Zallot C, Peyrin-Biroulet L. Deep remission in inflammatory bowel disease: looking beyond symptoms. Curr Gastroenterol Rep 2013;15:315. doi:10.1007/s11894-013-0315-7

10. Geboes K, Riddell R, Ost A, Jensfelt B, Persson T, Löfberg R. A reproducible grading scale for histological assessment of inflammation in ulcerative colitis. Gut 2000;47:404-409. doi:10.1136/gut.47.3.404

11. Cushing KC, Tan W, Alpers DH, Deshpande V, Ananthakrishnan AN. Complete histologic normalisation is associated with reduced risk of relapse among patients with ulcerative colitis in complete endoscopic remission. Aliment Pharmacol Ther 2020;51:347-355. doi:10.1111/apt.15568

12. D’haens G, Van Deventer S, Van Hogezand R, et al. Endoscopic and histological healing with infliximab anti-tumor necrosis factor antibodies in Crohn's disease: A European multicenter trial. Gastroenterology 1999;116:1029-1034. doi:10.1016/s00165085(99)70005-3

13. Wiernicka A, Szymanska S, Cielecka-Kuszyk J, Dadalski M, Kierkus J. Histological healing after infliximab induction therapy in children with ulcerative colitis. World J Gastroenterol 2015;21:10654-10661. doi:10.3748/wjg.v21.i37.10654

14. Yarur AJ, Jain A, Hauenstein SI, et al. Higher Adalimumab Levels Are Associated with Histologic and Endoscopic Remission in Patients with Crohn's Disease and Ulcerative Colitis. Inflamm Bowel Dis 2016;22:409415. doi:10.1097/MIB.0000000000000689

15. Christensen B, Hanauer SB, Gibson PR, Turner JR, Hart J, Rubin DT. Segmental Histological Normalisation Occurs in Ulcerative Colitis but Does Not Improve Clinical Outcomes. J Crohns Colitis 2020;14:13451353. doi:10.1093/ecco-jcc/jjaa068

16. Tursi A, Principi M, Picchio M, et al. Persistence of endoscopic rectal inflammation in ulcerative colitis treated with infliximab is not linked to ineffective TNFa downregulation. Gut 2016;65:539-540. doi:10.1136/ gutjnl-2015-310284

17. Leal RF, Planell N, Kajekar R, et al. Identification of inflammatory mediators in patients with Crohn's disease unresponsive to anti-TNFa therapy. Gut 2015;64:233-242. doi:10.1136/gutjnl-2013-306518 